\title{
L'ingénieur hydraulicien et les métiers de l'eau potable
}

\author{
P. Schulhof \& M. Vullierme \\ Compagnie générale des Eaux, Paris
}

\begin{abstract}
Depuis leur origine, les métiers de l'eau ont considérablement évolué. Cette évolution, d'abord lente, s'est accélérée progressivement à partir de la deuxième moitié du $\mathrm{XIX}^{\mathrm{e}}$ siècle lorsque, avec le développement démographique, économique et industriel, les besoins en eau se sont accrus et les problèmes de pollution sont devenus de plus en plus préoccupants.
\end{abstract}

Afin de préserver la qualité des ressources et de maintenir une répartition équitable entre les différents usagers, un «droit de l'eau » s'est constitué au fil des ans à partir de lois et de réglementations diverses. Cependant, le manque de concertation et l'absence d'une réelle action administrative coordonnée ne permettait pas, jusqu'à une époque récente, de le faire véritablement respecter.

La Loi sur l'Eau et la création des Agences financières de bassin marquent, au début des années 60 , un tournant très important. Les changements apportés comportent deux aspects essentiels :

- la gestion des eaux fait l'objet d'une planification rigoureuse qui prend en compte tous les éléments susceptibles d'influer sur la qualité des ressources : pollution urbaine, industrielle, agricole, contexte géographique, conditions climatiques,

- cette gestion s'appuie sur une approche économique où les utilisateurs de l'eau sont responsabilisés financièrement : les pollueurs et les utilisateurs payent une redevance, les stations de traitement et d'épuration sont subventionnées.

La mise en place de ces structures nouvelles a concrétisé l'évolution des métiers de l'eau potable en leur fixant un cadre commun. C'est dans ce cadre que doivent être envi- sagées les tâches et les responsabilités qui sont confiées à l'Ingénieur Hydraulicien.

Aujourd'hui, sa principale fonction est de concevoir des unités de traitement capables de produire en permanence et au moindre coût, une eau dont la qualité doit répondre à des normes de potabilité de plus en plus strictes.

Cette activité s'exerce en collaboration étroite avec des checheurs de nombreuses autres disciplines: sa démarche d'hydraulicien est orientée vers la mise en œuvre de procédés complexes, ce qui exige de dialoguer en permanence avec les spécialistes qui les ont définis. Le dialogue doit aussi être permanent avec les exploitants des usines de traitement, dans le souci de simplifier et d'automatiser la conduite des unités de production, de minimiser la maintenance et de favoriser une plus grande souplesse dans la gestion des installations. Enfin la notion de service public est pour lui fondamentale, elle le conduit à privilégier la fiabilité, la sécurité et la qualité.

Mais ces différents aspects sont eux-mêmes étroitement connectés puisque la prise en compte de l'exploitant et du consommateur doit se faire dès les premières étapes de la conception, de même que les contraintes de l'exploitation résultent des exigences d'un service public vital pour ses usagers.

Bien qu'elle conduise à une division quelque peu abstraite, c'est la démarche "du fil de l'eau» que nous avons adoptée pour cette présentation du métier de concepteur qui est celui de l'Ingénieur Hydraulicien :

- l'amont ou la réflexion préalable,

- l'usine ou la mise en cuvre sur le site,

- l'aval ou le souci du consommateur.

\section{The hydraulic engineer and drinking water activities}

Almost thirty years ago, as a result of a strong growth in the demand for water and the need to take measures against an increasing rate of pollution due to industrial and urban development, it became necessary to plan water resources management upon economic grounds. In 1964, this resulted in the French Water Act and the creation of Financial Water Authorities. Since then, with the merging of advanced technologies, drinking water activities have experienced tremendous changes. In this demanding context, the job of the hydraulics engineer has also drastically changed. 


\section{L'amont ou la réflexion préalable}

La conception des usines de traitement d'eau potable requiert en premier lieu une très bonne connaissance de l'évolution récente des technologies et des matériels.

En ce qui concerne la qualité de l'eau, la définition de nouvelles normes suit de près les progrès des méthodes analytiques mises en œuvre dans les laboratoires. Le dosage de substances précédemment indécelables, appelle leur contrôle tout au long de la chaîne de traitement et la définition de procédés nouveaux pour les éliminer ou les inactiver: on suit par exemple l'évolution de la matière organique dans la filière et dans le réseau de distribution, on élimine des molécules de l'ordre du nanomètre comme les pesticides, on mesure précisément l'efficacité des procédés de stérilisation, etc... Ces traitements performants, qui font appel à des disciplines très variées (mécanique des fluides, chimie, biologie, biochimie), sont d'abord mis au point dans des laboratoires de recherche, à l'échelle de pilotes.

Dans cette première phase le rôle de l'ingénieur hydraulicien est fondamental : il doit faire une bonne analyse du procédé afin de dégager les points qui nécessiteront une approche spécifique au plan de l'hydraulique. Son action est aussi rétroactive : il oriente les essais qui sont réalisés, pour que les traitements mis au point soient compatibles avec une mise en œuvre à l'échelle industrielle.

Le procédé étant défini, la transition entre le pilote et l'usine de traitement exige de bien analyser les phénomènes mis en jeu, afin de concevoir des ouvrages qui respectent les grandeurs caractéristiques des réactions et des process. A titre d'exemples:

- une cuve de désinfection par l'ozone doit permettre de respecter un temps de contact pour que puisse s'effectuer une complète inactivation des virus,

- un procédé d'injection de réactif coagulant doit assurer une dispersion du produit au sein de l'eau à traiter dans un laps de temps inférieur au temps caractéristique de la réaction d'hydrolyse,

- la vitesse de passage des filets fluides dans un ouvrage de décantation doit être suffisamment faible pour respecter la chute des matières en suspension.

L'automatisation des installations est un autre aspect important de l'évolution technologique des usines de traitement d'eau. Pour cette raison, l'ingénieur hydraulicien est en contact permanent avec les spécialistes des automatismes: le matériel et la configuration hydraulique doivent être choisis de telle sorte qu'un mode de régulation simple, prenant en compte les temps de réponse des appareils, puisse être mis en place. D'autre part, une description très précise des différents modes de fonctionnement de l'installation projetée (mise en route, phases transitoires de changement de régime, arrêt), mentionnant l'ordre et la durée des opérations successives, doit être fournie aux ingénieurs en charge des automatismes afin que tous les paramètres qui conditionnent la conduite de l'ouvrage soient pris en compte dans l'automatisation.

Enfin, dès cette phase initiale de la conception des ouvrages, se pose le problème du choix des équipements : dans ce domaine aussi, l'évolution des techniques est extrêmement rapide. L'ingénieur hydraulicien doit être en contact avec les fabricants de matériels afin de se tenir informé des produits nouveaux, de leurs performances et de leurs conditions d'utilisation :

- le choix d'une pompe d'injection de produit chimique doit prendre en compte les problèmes possibles de corrosion, la quantité de réactif à doser, la stabilité chimique du composé utilisé,

- le choix d'une vanne permet de privilégier certains aspects d'un écoulement : perte de charge minimale, homogénéisation des filets fluides, régulation de débit, etc...

D'autre part, dans la gamme de matériel permettant d'atteindre un objectif donné, ce choix doit bien sûr se porter sur l'équipement qui permet de minimiser les coûts d'investissement et d'entretien.

Ainsi, l'ingénieur hydraulicien doit posséder une vaste culture scientifique et technique, qui lui permette de travailler en concertation avec des spécialistes de nombreux domaines et de choisir, pour la mise en œuvre d'un procédé, le matériel le plus moderne et le plus performant.

\section{L'usine ou la mise en æuvre sur le site}

L'ingénieur hydraulicien doit aussi accorder une très grande importance à la mise en œuvre du projet sur le site. Il doit tenir compte des contraintes spécifiques d'environnement, des modes de fonctionnement privilégiés de l'usine, des impératifs de production et de maintenance.

La première notion fondamentale est celle de l'environnement au sens large, c'est-à-dire du contexte de réalisation du projet.

Dans le cas le plus favorable de la réalisation d'une unité de production indépendante, où les solutions technologiques sont relativement nombreuses, c'est la capacité de l'usine qui conditionne sa conception: une unité qui produit $20000 \mathrm{~m}^{3} / \mathrm{j}$ doit être pensée différemment d'une unité susceptible de produire $800000 \mathrm{~m}^{3} / \mathrm{j}$. Le débit nominal induit une corrélation entre le nombre et la taille des ouvrages, ainsi :

- la construction d'un ensemble de filtration capable de traiter un débit important peut conduire à la réalisation de filtres de grande surface, ce qui est impossible pour une unité de petite taille, le lavage d'un filtre devenant alors incompatible avec le maintien d'un débit minimum de production,

- une unité de gros débit doit être conçue pour fonctionner en continu car son secours est beaucoup plus difficile que celui d'une petite usine. Ainsi tous les ouvrages doivent pouvoir être entretenus sans interruption de production, ce qui implique de doubler systématiquement toutes les canalisations et de pouvoir isoler au moins une file sur chaque ouvrage, permettant ainsi de produire un débit, certes inférieur au débit nominal, mais limitant les demandes d'aide extérieure. 
S'il s'agit au contraire de l'insertion ou de la modification d'un ouvrage dans une filière déjà existante, la liberté de choix est alors plus réduite, et ce sont alors souvent les problèmes d'encombrement et de ligne piézométrique qui conditionnent la conception :

- étant donné le prix élevé des terrains dans les zones urbanisées, un effort particulier devra porter sur la réalisation d'ouvrages compacts, ménageant au maximum des possibilités d'évolution ultérieure de l'unité de traitement, - selon les cas, afin de compenser l'énergie dissipée dans l'installation nouvelle, il faudra choisir entre une modification des ouvrages amont et aval ou la construction d'une nouvelle station de pompage. Cette deuxième solution, qui nécessite la construction d'un réservoir de pompage et accrôt la complexité de la régulation, devra dans la mesure du possible être évitée: on cherchera donc lors de la conception à minimiser les pertes de charge dues au nouvel ouvrage.

L'ingénieur hydraulicien doit aussi prendre en compte tous les paramètres géographiques et climatiques pouvant influer sur la conception des ouvrages; on peut citer par exemple :

- le niveau de la nappe phréatique qui peut conduire à modifier l'assiste d'un bâtiment ou à renforcer un fond de réservoir qui pourrait être mis en pression par une élévation du niveau de la nappe,

- la cote maximale de la rivière qui conditionne la hauteur des trop-pleins de sécurité,

- l'importance des précipitations atmosphériques qui conduit, le cas échéant, à couvrir certains ouvrages,

- les valeurs des températures extrêmales, nécessitant la mise hors-gel ou le refroidissement de certaines installations, la ventilation des chambres de vannes.

Enfin, toujours dans le cadre de la prise en compte de l'environnement, la présentation architecturale est un élément important de la conception. En effet, dans la grande majorité des cas, le traitement des eaux de surface se fait à proximité des zones urbanisées desservies: les unités de production doivent donc s'intégrer parfaitement dans le site retenu. Outre le respect du plan d'occupation des sols, qui est impératif et peut avoir des incidences sur l'aspect hydraulique, un travail de collaboration doit s'engager très tôt avec un architecte de bâtiments industriels afin que l'aspect extérieur des ouvrages permette une insertion harmonieuse dans l'environnement. De même, l'aménagement intérieur doit être fonctionnel et agréable. En particulier, les axes de passage et les ouvertures doivent être conçus de telle sorte que les éléments importants du traitement soient mis en valeur et que le mode de fonctionnement de l'installation puisse être rapidement appréhendé.

La seconde notion fondamentale est celle de l'exploitation et, corrélativement, de la maintenance. L'ouvrage conçu doit être fonctionnel, facile à gérer et à entretenir. Dans ce cadre, l'ingénieur hydraulicien doit dialoguer en permanence avec l'exploitant ; c'est d'ailleurs souvent l'expérience de ce dernier qui permettra de définir de manière optimale un certain nombre de paramètres de l'installation. Sans prétendre à l'exhaustivité, on peut citer les points suivants qui doivent faire l'objet d'une attention particulière :

- la capacité maximale de l'usine est une donnée qu'il importe de compléter par la définition de plusieurs modes privilégiés de fonctionnement: le dimensionnement et le nombre des ouvrages doivent permettre une gestion aisée pour chacun de ces modes,

- la fiabilité de l'exploitation conduit à prévoir la mise en place de matériel de secours et à laisser une marge de sécurité dans le calcul des installations,

- l'exploitation doit être souple, particulièrement dans les phases transitoires que sont les changements de régime : il convient donc par exemple de prévoir des volumes tampons suffisants pour compenser le temps de réaction des organes de régulation, de mettre en place des bipasses permettant d'intervenir sur un ouvrage sans immobiliser l'ensemble de la filière,

- la conception doit prendre en compte les problèmes de maintenance. Cet aspect conduit notamment à restreindre la variété des matériels choisis de manière à limiter les stocks de rechange, à prévoir des accès faciles dans les bâtiments pour le remplacement des équipements, à mettre en place dès l'origine des moyens de manutention appropriés (ponts roulants,...),

- enfin, l'ingénieur hydraulicien doit être à l'écoute des problèmes rencontrés sur les ouvrages déjà en place. Il effectue un suivi des installations et suggère des améliorations de leurs conditions de fonctionnement.

Homme de dialogue, l'ingénieur hydraulicien est donc aussi homme de terrain qui sait, lors de la conception d'un projet, apprécier le contexte de réalisation des ouvrages et prendre en compte les contraintes d'exploitation spécifiques aux usines de traitement d'eau potable.

\section{L'aval ou le souci du consommateur}

Pour compléter la liste des différents aspects du travail de conception, il est nécessaire de souligner encore une fois l'objectif premier du traiteur d'eau : délivrer au consommateur une eau de qualité irréprochable, en quantité suffisante, au meilleur coût.

Ayant mis en place des ouvrages de traitement adaptés, l'hydraulicien doit prévoir des moyens de contrôler la quantité et la qualité au sein des usines et dans le réseau de distribution.

Quantitativement, dans les usines de traitement, il est impératif que l'exploitant connaisse à tout moment le débit circulant dans les différents ouvrages. Une bonne connaissance de l'instrumentation doit donc permettre à l'ingénieur hydraulicien de choisir et de positionner des capteurs (vitesse, pression différentielle) qui donneront au poste de commande de l'usine des informations fiables concernant les débits. Pour ce faire, des longueurs droites de canalisations doivent être ménagées afin d'obtenir des écoulements stabilisés. 
Qualitativement, à différentes étapes du traitement et dans le réseau, certains paramètres physico-chimiques doivent être relevés en continu ou périodiquement : le $\mathrm{pH}$, la température, la concentration en matières organiques, la concentration en ozone, la turbidité, etc... L'hydraulicien prévoit donc des points de prélèvement en s'assurant que les échantillons recueillis reflètent correctement la réalité. La bonne mise en œuvre de ce type d'équipement exige encore une fois une bonne connaissance des moyens d'instrumentation, ainsi que des notions de physico-chimie de l'eau.

En aval de l'usine, le réseau de distribution doit aussi faire l'objet d'études de conception très poussées. Un réseau mal dimensionné peut être responsable d'une dégradation du service rendu au consommateur :

— un réseau sous-dimensionné ne permet pas de délivrer une pression suffisante à tous les abonnés,

- un réseau peu maillé est difficilement secourable en cas d'incident sur une de ses branches,

- la présence de zones mortes favorise les développements de micro-organismes.

Le réseau de distribution fait actuellement l'objet de nombreuses études: l'objectif est d'améliorer sa qualité (résistance à la corrosion, optimisation des écoulements) et de développer des outils qui permettront de mieux contrôler les phénomènes hydrauliques, physico-chimiques et bactériologiques mis en jeu. Dans ce domaine, l'ingénieur hydraulicien utilise l'outil informatique, pour modéliser les écoulements dans le réseau et les interactions entre l'hydraulique et l'évolution de la qualité de l'eau.

Le souci d'assurer un service public de qualité est indissociable de la notion du respect de l'environnement: le producteur d'eau ne peut être un pollueur. Lors de la conception de usines de production d'eau potable, il est donc indispensable de concevoir des ouvrages permettant de retraiter les effluents générés par le traitement de l'eau : boues issues des ouvrages de décantation, eau de lavage des filtres, etc... De même tous les rejets en rivière doivent être conçus pour recevoir un ensemble d'appareils de mesure permettant de contrôler en permanence les principales caractéristiques des fluides rejetés. Cette notion d'industrie "propre ", qui est amenée dans l'avenir à prendre une importance considérable, est un élément important de la crédibilité des traiteurs d'eau vis-à-vis des usagers.
Enfin, le souci du consommateur exige d'insister encore sur l'aspect économique sous-jacent au travail de conception effectué par l'ingénieur hydraulicien. En premier lieu, le coût d'investissement est une donnée importante ; pour la mise en œuvre d'un procédé, il faut rechercher toutes les solutions permettant d'assurer une bonne efficacité du traitement et savoir les chiffrer rapidement. Mais il faut aussi pouvoir évaluer avec une bonne précision le coût d'exploitation des ouvrages et la durée d'amortissement de ceux-ci, car c'est la synthèse de toutes ces données qui lui permettra en dernier ressort de choisir la solution correspondant à l'optimum économique.

Ce dernier volet du travail de l'ingénieur hydraulicien en souligne donc deux aspects: le contrôle rigoureux du produit délivré et l'optimisation de son coût, afin de garantir à l'usager la meilleure qualité de service.

\section{Conclusion}

Au sein des métiers de l'eau potable, la profession d'ingénieur hydraulicien a donc considérablement évolué au cours des dernières décennies. Cette brève description des tâches et des responsabilités qui lui sont confiées montre qu'au-delà des connaissances techniques qui sont nécessaires dans des domaines de plus en plus variés, c'est véritablement une ouverture d'esprit qui caractérise le mieux ce qu'est devenu aujourd'hui ce métier.

D'abord un spécialiste des problèmes d'écoulements hydrauliques, l'ingénieur hydraulicien qui travaille dans le domaine de l'eau potable doit aussi être un bon généraliste. C'est un homme de réflexion, ouvert au dialogue avec les chercheurs ; c'est un homme de terrain, en contact avec les exploitants ; c'est un homme de service public, soucieux du consommateur et de son environnement, capable dans son travail de conception de faire la synthèse d'un grand nombre de paramètres scientifiques, techniques et économiques.

A la veille de l'Europe de 1993, il est de la responsabilité des traiteurs d'eau d'aider à définir des formations appropriées, afin que des ingénieurs compétents soient capables d'exporter les technologies et les méthodes françaises en matière de traitement d'eau potable. 